\title{
Synthèse et évaluation de l'activité pharmacologique de la 4-phényl-1,5- benzodiazépin-2-one et ses dérivés
}

\author{
P.M. KANYONGA ${ }^{1 *}$, A. ZELLOU ${ }^{2}$, M. ESSASSI $^{1}$ et Y. CHERRAH $^{2}$ \\ ${ }^{1}$ Laboratoire de Chimie Organique Hétérocyclique, Pôle de Compétences Pharmacochimie, Faculté des \\ Sciences- Agdal, BP :1014, Avenue : Ibn Battouta / Rabat-Maroc. \\ ${ }^{2}$ Laboratoire de Pharmacologie et Toxicologie, Faculté de Médecine et Pharmacie, Rabat - Instituts, Maroc. \\ *Auteur correspondant, E-mail: manuelekanyonga@yahoo.fr
}

\section{RESUME}

La synthèse et l'étude de la réactivité de la 4-phényl-1,5-benzodiazépin-2-one nous a permis d'obtenir deux dérivés: la 4-phényl-1,3,4,5-tétrahydro-1,5-benzodiazépin-2-one et la 4-phényl-1,5-benzodiazépin-2thiole. Ces produits ont fait l'objet d'une étude toxicologique et nous avons aussi exploré leur activité sur le système nerveux central, notamment l'action analgésique, grâce à un test spécifique. Après analyse de nos résultats, nous avons constaté que les composés testés s'avèrent atoxiques à des doses thérapeutiques, avec une $\mathrm{DL}_{50}$ supérieure à $1000 \mathrm{mg} / \mathrm{kg}$ par voie intrapéritonéale, et exercent une action analgésique modérée, surtout pour les composés 1 et 3 vis-à-vis de l'acide acétylsalicylique. Le produit 2 a une action analgésique moins importante.

(c) 2010 International Formulae Group. All rights reserved.

Mots clés : 1,5-benzodiazépine, synthèse, activités pharmacologiques, toxicité aiguë, activité analgésique.

\section{INTRODUCTION}

Un examen de la littérature montre que depuis les premiers travaux sur la famille des benzodiazépines, ces dernières ont fait l'objet de nombreuses études et ceci pour l'intérêt qu'ils présentent, notamment sur le plan pharmacologique (Beaulieu, 2006; Tosti et al., 2007) et chimique (Ahabchane et al., 1999). Leur activité sur le système nerveux central est la plus étudiée mais, les opportunités que ces molécules offrent en tant que matières premières pour la synthèse des substances actives pouvant être utilisées à d'autres fins sont intéressantes [Activité antibactérienne (Zniber et al., 1991); activité antitumorale (Kuo-Hsing et Thoshiro, 1978); activité phytopharmaceutique et agro-vétérinaire, etc.], et le fait que leur synthèse utilise souvent des méthodes nécessitant la mise en jeu de réactifs onéreux voire même dangereux (Kirkiacharian, 1996; Graham et Depovere,
2002). C'est ainsi que nous nous sommes fixés comme objectif de synthétiser les dérivés de la 4-phenyl-1,5-benzodiazépin-2one en utilisant des réactifs usuels, facilement accessibles et d'explorer leur toxicité et leur activité analgésique par comparaison avec une substance de référence. Ce qui permettra de dire si la 4-phenyl-1,5-benzodiazépin-2-one et ses dérivés synthétisés ont une action analgésique périphérique à des doses thérapeutiques, car de nombreux auteurs préconisent l'utilisation de benzodiazépines comme analgésiques secondaires ou comme co-analgésiques (Aveline et al., 2001; Muster et Ben Slama, 2004).

\section{MATERIEL ET METHODES \\ Produits}

La 4-phényl-1,5-benzodiazépin-2-one a été synthétisée par condensation de l'Ophénylenediamine avec le benzoylacétate 
d'éthyle dans le xylène pendant une heure (Essassi et Fifani, 1987 ; Zellou et al., 1998a). Le produit obtenu a été soumis aux réactions d'hydrogénation catalytique en présence de palladium sur charbon et de sulfuration par le pentasulfure de phosphore dans la pyridine (Essassi et Fifani, 1987; Sidya et al., 2004), ce qui a permis d'obtenir: la 4-phényl-1,3,4,5tétrahydro-1,5-benzodiazépin-2-one et la 4phényl- 1,5-benzodiazépin-2-thiole dont les structures sont présentées sur la Figure 1.

Les points de fusion non corrigés ont été mesurés à l'aide du banc chauffant Köffler. Les spectres de résonance magnétique nucléaire $\left({ }^{1} \mathrm{H},{ }^{13} \mathrm{C}\right)$ ont été enregistrés sur un appareil AVANCE 300 Bruker opérant à $300 \mathrm{MHz}$, en solution dans le chloroforme deutérié. Les déplacements chimiques sont donnés en ppm par rapport à la référence interne TMS. Les spectres de masse ont été effectués par impact électronique à l'aide de l'appareil VARIAN MAT 311A. Les spectres IR ont été enregistrés en film de $\mathrm{KBr}$, sur un spectromètre IR-TF VERTEX 70 entre 600 et $4000 \mathrm{~cm}^{-1}$.

L'obtention de ces produits se fait selon les modes opératoires ci-dessous :

- 4-phényl-1,5-benzodiazépin-2-one : mettre dans un ballon de $250 \mathrm{ml}, 0,01$ mole d'orthophénylène-diamine et 0,011 mole de benzoylacètate d'éthyle dans $100 \mathrm{ml}$ de xylène, porter à reflux pendant 1 heure, et refroidir. On obtient un précipité, filtré et lavé avec de l'éthanol, puis séché et on obtient une poudre de couleur blanche.

-4-phényl-1,3,4,5-tétrahydro-1,5-benzodiazépin-2-one : mettre dans un ballon de $100 \mathrm{ml}$ 0,005 mole de la 4-phényl-1,5-benzodiazépin2-one, ajouter une pincée de $\mathrm{Pd}-\mathrm{C}$ dans $60 \mathrm{ml}$ d'éthanol sous pression d'hydrogène Apres 48 heures, filtrer la solution obtenue, la concentrer à sec et la recristalliser dans l'éthanol, on obtient une poudre de couleur blanche.

- 4-phényl-1,5-benzodiazépin-2-thiole : mettre dans un ballon de $250 \mathrm{ml}, 0,01$ mole de la 4phényl-1,5-benzodiazépin-2-one, 0,03 mole de $\mathrm{P}_{2} \mathrm{~S}_{5}$ et $60 \mathrm{ml}$ de la pyridine, porter à reflux pendant 1 heure, puis concentrer et laver le résidu avec l'eau chaude et sécher. On obtient une poudre de couleur grise.
Ces structures ont été élucidées sur la base des données spectrales: R.M.N ${ }^{1} \mathrm{H}$; R.M.N ${ }^{13}$ C; I.R ; Spectroscopie de masse et les paramètres physicochimiques sont repris dans le Tableau 1.

\section{Etude pharmacologique \\ Produits testés}

1: 4-phényl-1,5-benzodiazépin-2-one;

2: 4-phényl-1,3,4,5-tetrahydro-1,5-benzodiazépin-2-one;

3: 4-phényl-1,5-benzodiazépin-2-thiole.

Animaux

L'étude est effectuée sur des souris swiss adultes, des deux sexes, provenant de l'élevage du Laboratoire de Pharmacologie de la Faculté de Médecine et de Pharmacie de Rabat. Leur poids se situe entre 20 et $30 \mathrm{~g}$, elles étaient maintenues dans les mêmes conditions de température $\left(25 \pm 2{ }^{\circ} \mathrm{C}\right)$, humidité relative située entre 40 à $70 \%$, et l'accès à l'eau et aux aliments à volonté. (Broustail, 1967; Laroche et Rousselet, 1990). L'utilisation des animaux a été faite en conformité avec les guides d'utilisation des animaux de Laboratoire (Anonyme, 1996), (Ernest, 1993).

\section{Toxicité aigü̈}

Elle est déterminée selon la méthode de Lichfield et Wilcoxon (Dupont, 1970; Boniface et al., 1972). Nous avons utilisé 6 lots de 10 souris. Les animaux de chaque lot reçoivent une dose unique des composés testés par voie intrapéritonéale sous forme d'une suspension dans la gomme arabique à $10 \%$. Les doses administrées par lot sont les suivantes: Lot 1: $100 \mathrm{mg} / \mathrm{kg}$, Lot 2: 200 $\mathrm{mg} / \mathrm{kg}$, Lot 3: $400 \mathrm{mg} / \mathrm{kg}$, Lot 4: $800 \mathrm{mg} / \mathrm{kg}$, Lot 5: $1600 \mathrm{mg} / \mathrm{kg}$, Lot 6: $2000 \mathrm{mg} / \mathrm{kg}$.

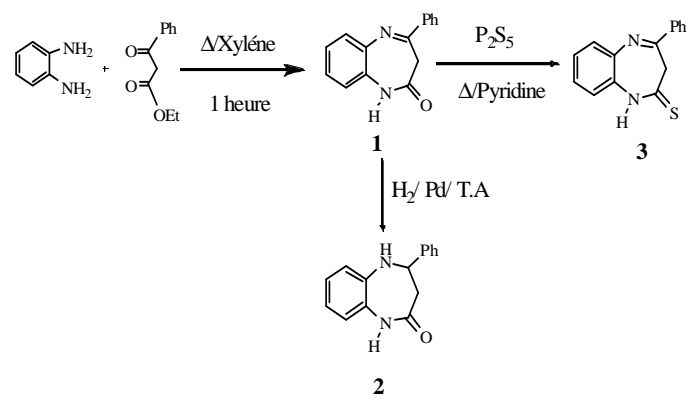

Figure 1: Schéma de la synthèse des produits. 
Tableau 1: Paramètres d'identification de la 4-phényl-1,5-benzodiazépin-2-one ; de la 4-phényl1,3,4,5-tetrahydro-1,5-benzodiazépin-2-one ; et de la 4-phényl-1,5-benzodiazépin-2-thiole.

\begin{tabular}{|c|c|c|c|}
\hline Produit & Rendement & $\begin{array}{l}\text { Point de } \\
\text { fusion }\end{array}$ & Données spectrales \\
\hline $\begin{array}{l}\text { 4-phényl-1,5benzo-di- } \\
\text { azépin-2-one }\end{array}$ & $90 \%$ & $\begin{array}{l}198-200{ }^{\circ} \mathrm{C} \\
\text { (Ethanol) }\end{array}$ & $\begin{array}{l}\text { * Spectre } \mathrm{RMN}{ }^{1} \mathrm{H}\left(\mathrm{CDCl}_{3}\right): 3,4 \mathrm{ppm}\left(2 \mathrm{H}, \mathrm{S}, \mathrm{CH}_{2}\right) ; 7,20-7,90 \mathrm{ppm} \\
(9 \mathrm{H} \text { aromatiques, m); } 9,37 \mathrm{ppm}(1 \mathrm{H}, \mathrm{s}, \mathrm{NH}) ; \\
\text { * Spectre } \mathrm{RMN} 13 \mathrm{C}: 39,79 \mathrm{ppm}(\mathrm{CH} 2) ; 121,82-130,98 \mathrm{ppm} \\
(\mathrm{CH} \text { aromatique); } 129,14 \mathrm{ppm}(\mathrm{C} \text { aromatique }) ; 137,62-139,91 \mathrm{ppm} \\
\text { (C aromatique); 158,64 ppm( } \mathrm{C}=\mathrm{N}) ; 167,80 \mathrm{ppm}(\mathrm{C}=\mathrm{O}) ; \\
\text { * Spectre IR: } \square_{\mathrm{C}=\mathrm{O}}: 1670 \mathrm{Cm}^{-1} ; \\
\text { * Spectre de masse }: \mathrm{M}^{+}(\mathrm{m} / \mathrm{z})=236\end{array}$ \\
\hline $\begin{array}{l}\text { 4-phényl-1,3,4,5-tétrahydro- } \\
\text { 1,5-benzodiazépin-2-one }\end{array}$ & $85 \%$ & $\begin{array}{c}176-178^{\circ} \mathrm{C} \\
\text { (éthanol) }\end{array}$ & $\begin{array}{l}\text { * Spectre } \mathrm{RMN}{ }^{1} \mathrm{H}: 7,10 \mathrm{ppm}(\mathrm{H} \text { aromatiques, } \mathrm{m}) \text {; un multiplet à } \\
5 \mathrm{ppm}(\mathrm{H} \text { sur le } \mathrm{C} 4) \text { et un multiplet centré à } 2,80 \mathrm{ppm} \text { ( } 2 \mathrm{H} \text { portés par } \\
\text { le groupe méthylène en position } 3 \text {.) } \\
{ }^{*} \text { spectre IR pris dans le } \mathrm{KBr} \text { montre la présence d'une bande à } \\
1640 \mathrm{Cm}^{-1} \text { attribuable à } \square_{\mathrm{C}=\mathrm{O}} \text { et la disparition de } \square_{\mathrm{C}=\mathrm{N}} \text {. } \\
\text { * spectre de masse : un pic moléculaire } \mathrm{M}^{+}(\mathrm{m} / \mathrm{z}): 238\end{array}$ \\
\hline $\begin{array}{l}\text { 4-phényl- 1,5-benzo- } \\
\text { diazépin-2-thiole }\end{array}$ & $80 \%$ & $222^{\circ} \mathrm{C}$ & $\begin{array}{l}\text { * Spectre RMN }{ }^{1} \mathrm{H}: \mathrm{CH}_{2}: 3,9 \mathrm{ppm} ; \mathrm{CH} \text { (aromatiques) : } \\
7,2-7,9 \mathrm{ppm} ; \\
\text { * Spectre de masse: } \mathrm{M}^{+}(\mathrm{m} / \mathrm{z}): 252 \\
\text { * Spectre IR : } \square_{\mathrm{C}=\mathrm{S}}: 1140 \mathrm{Cm}^{-1}\end{array}$ \\
\hline
\end{tabular}

Légende: $\mathrm{d}=$ doublet $; \mathrm{t}=$ triplet $; \mathrm{q}=$ quadruplet $; \mathrm{m}=$ massif .

Les animaux ont reçu un volume de 0,4 $\mathrm{ml}$ pour $20 \mathrm{~g}$ de poids corporel, et ils étaient gardés en observation pendant 14 jours.

\section{Etude de l'activité analgésique}

Elle est réalisée par l'intermédiaire du test de «KOSTER» (Cherrah et al., 2007) qui consiste à rechercher une éventuelle protection vis-à-vis des crampes induites par l'injection intrapéritonéale de l'acide acétique. Ce test permet d'explorer l'action analgésique périphérique des produits.

Pour ce faire, les animaux sont subdivisés en 3 lots de 5 animaux (souris):

- Le lot 1 témoin recevant le véhicule des produits ;

- Le lot 2 traité par les produits testés,

- Le lot 3 de référence recevant l'acide acétylsalicylique à la dose de $100 \mathrm{mg} / \mathrm{kg}$.

Les animaux traités reçoivent 100 $\mathrm{mg} / \mathrm{kg}$ par voie intrapéritonéale des composés à tester, cette dose est fixée sur la base de nos résultats de toxicité aiguë. Et le comptage du nombre de crampes débute au moment de l'injection et se termine vingt minutes après. Le pourcentage de protection est calculé selon la formule suivante :

$\underline{\text { Nombrelecrampedulot témoin- Nombrelecrampesdulot traité }} \times 100$ Nombreslecrampedulot témoin

\section{Analyses statistiques}

Les résultats ont été exprimés en moyenne \pm écartype, et ces moyennes ont été comparées grâce au test ANOVA, et les résultats sont considérés comme significatifs au $\mathrm{p}<0,05$.

\section{RESULTATS \\ Toxicité aiguë}

Dans nos conditions de travail, nous avons obtenu les valeurs suivantes : pour les trois produits : $1617,08 \mathrm{mg} / \mathrm{kg}$ pour le produit $1,1219,20 \mathrm{mg} / \mathrm{kg}$ pour le produit 2 et 596,50 $\mathrm{mg} / \mathrm{kg}$ pour le produit 3. Le Tableau 2 présente les DL ${ }_{50}$ des trois produits ainsi que leurs limites de confiance à $95 \%$. 
Tableau 2: $\mathrm{DL}_{50}$ de la 4-phényl-1,5-benzodiazépin-2-one ; de la 4-phényl-1,3,4,5-tetrahydro-1,5benzodiazépin-2-one ; et de la 4-phényl-1,5-benzodiazépin-2-thiole.

\begin{tabular}{lcc}
\hline & $\begin{array}{c}\text { Voie intrapéritonéale } \\
{[\mathbf{m g} / \mathbf{K g}]}\end{array}$ & Limites de confiance à 95\% \\
\hline 1: 4-phenyl-1,5-benzodiazepin-2-one & 1617,08 & $1450,34<\mathrm{DL} 50<1803$ \\
2:4-phenyl-1,3,4-tetrahydro- & 1219,20 & $1113,49<\mathrm{DL} 50<1334,98$ \\
$\begin{array}{l}\text { 1,5 benzodiazepin-2-one } \\
\text { 3: 4-phenyl-1,5-benzodiazepin-2-thiole }\end{array}$ & 596,50 & $464,80<\mathrm{DL} 50<724,3$ \\
\hline
\end{tabular}

\section{Suivi Pondéral}

Le suivi de l'évolution pondérale des animaux durant quinze jours montre de manière générale une diminution du poids corporel durant les trois premiers jours. Passé ce délai, ce poids augmente normalement et devient supérieur au poids initial de l'animal traité (Figure 2).

\section{Activité analgésique}

Les Souris traitées par les produits $(1,2$ et 3) ont développé un nombre de crampes inférieures à celles des souris témoins, soit $26 \pm 2$ représentant $40,9 \%$ de protection pour le produit $1 ; 38 \pm 3$ représentant $13,6 \%$ de protection pour le produit 2 et $31 \pm 2$ représentant $29,5 \%$ pour le produit 3 . Alors que les souris du groupe de référence ont développé un nombre réduit de crampes $(17 \pm 4$ de crampes en 20 minutes, ce qui correspond à $61,4 \%$ de protection) (Tableau 2).

\section{DISCUSSION}

Dans nos conditions expérimentales, la 4-phényl-1,5-benzodiazépin-2-one et ses dérivés testés s'avèrent atoxiques, avec une $\mathrm{DL}_{50}$ supérieure à $1000 \mathrm{mg} / \mathrm{kg}$ pour les produits 1 et 2 , alors que le produit 3 a présenté une toxicité moyenne par voie intrapéritonéale. $\mathrm{La}^{\mathrm{DL}} \mathrm{L}_{50}$ du produit 3 peut s'expliquer par la présence du soufre dans la molécule. Nos résultats confirment ceux de la littérature qui considèrent les benzodiazépines comme des substances à faible toxicité (Laroche et al., 1986; Lullmann et al., 2001) et aussi les résultats obtenus par Zellou et al. (1998a; 1998b); Chammach et al. (2001) sur les substances de cette famille. Au regard de l'évolution pondérale des animaux, nous avons observé une diminution pondérale dans les 72 heures après administration des produits (soit $24,7 \pm 1,85 \mathrm{~g}$ au $\mathrm{J} 1$ passant à $22,44 \pm 1,68 \mathrm{~g}$ au J3 , puis $23,07 \pm 2,39 \mathrm{~g}$ au J4 et atteint $27,32 \pm 1,9 \mathrm{~g}$ au J14 pour le produit 1 ; $24,34 \pm 1,12 \mathrm{~g} \mathrm{~J} 1$ passant à $22,48 \pm 2,7 \mathrm{~g}$ au J3, puis $23,32 \pm 2,3 \mathrm{~g}$ au $\mathrm{J} 4$ et atteint $25,51 \pm 2,09 \mathrm{~g}$ au J14 pour le produit $2 ; 25,6 \pm 2,4 \mathrm{~g}$ au $\mathrm{J} 1$ passant à 20,48 $\pm 2,7 \mathrm{~g}$ au J3, puis $22,32 \pm 2,3 \mathrm{~g}$ au J4 et atteint $27,00 \pm 2,09 \mathrm{~g}$ au J14 pour le produit 3), ceci peut être dû à l'effet sédatif des produits, qui réduirait la consommation par les animaux de la nourriture et l'eau.

Au regard des résultats de l'évaluation de l'activité analgésique, nous avons constaté que les souris témoins ont développé un nombre important de crampes (44 crampes pendant 20 minutes) et de façon progressive après administration de l'acide acétique, ceci est remarqué au début par un étirement des pattes postérieures et une contraction des muscles intercostaux précédents l'apparition des crampes franches.

Les souris traitées par l'acide acétylsalicylique ont développé un nombre réduit de crampes par rapport au lot témoin et aux animaux traités par nos produits. Ceci est confirmé par le pourcentage de protection observé $(61,4 \%)$, alors que les souris traitées par les produits synthétisés ont développé un nombre réduit de crampes par rapport aux souris témoins ne recevant que le solvant. En effet, les pourcentages de protection vis-à-vis des crampes calculés pour les différents produits montrent une supériorité relative du composé $1(40,9 \%)$ suivi du produit 3 $(29,5 \%)$; alors que le produit $2(13,6 \%)$ présente une action inférieure, ceci montre que la présence de substituant (sulfuration et hydrogénation) sur la molécule de départ 1 entraîne une diminution de l'activité 


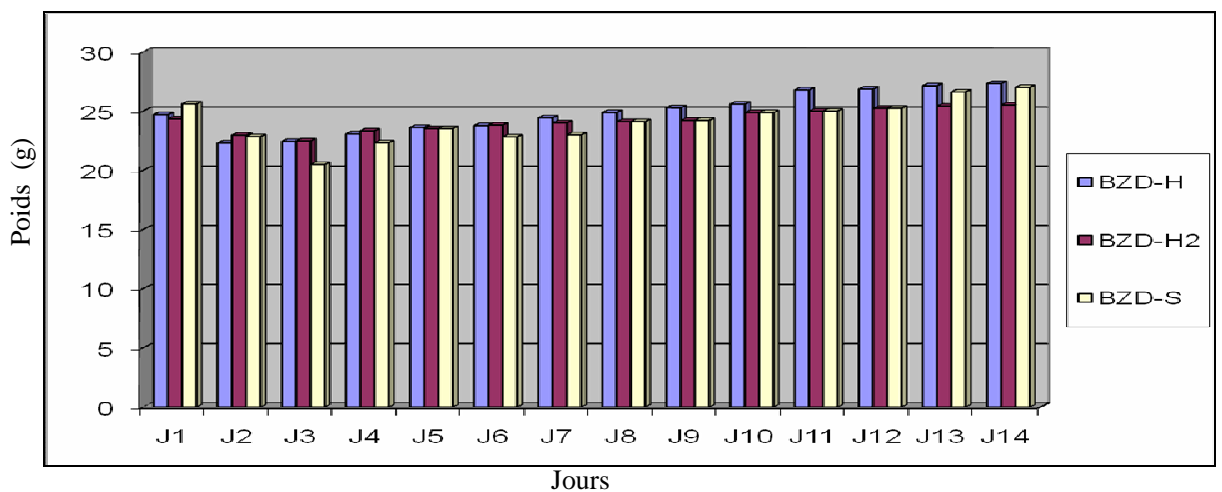

Figure 2: Evolution pondérale des animaux traités avec la 4-phényl-1,5-benzodiazépin-2one, 4-phényl-1,3,4-tetrahydro-1,5 benzodiazépin-2-one, 4-phényl-1,5-benzodiazépin-2thiole. BZD-H: Produit 1; BZD-H2: Produit 2 ; BZD-S: Produit 3.

Tableau 3: Effets de la 4-phényl-1,5-benzodiazépin-2-one, de la 4-phényl-1,3,4,5tetrahydro-1,5-benzodiazépin-2-one, de la 4-phényl-1,5-benzodiazépin-2-thiole et de l'acide acétylsalicylique sur le nombre de crampes abdominales induites par l'acide acétique chez la souris.

\begin{tabular}{lcc}
\hline Produits & $\begin{array}{c}\text { Nombre de crampes abdominales } \\
\text { en 20 minutes }\end{array}$ & $\begin{array}{c}\text { Inhibition des crampes } \\
\text { abdominales (\%) }\end{array}$ \\
\hline 1 & $26 \pm 2$ & 40,9 \\
2 & $38 \pm 3$ & 13,6 \\
3 & $31 \pm 2$ & 29,5 \\
AAS & $17 \pm 4$ & 61,4 \\
\hline
\end{tabular}

analgésique. Ces résultats montrent que ces produits n'ont pas une activité semblable à celle de l'A.A.S $[\mathrm{F}(3,16)=195$ à $\mathrm{p}<0,05]$.

Ces résultats nous permettent de confirmer les propos d'Aveline et al. (2001) qui préconisent la prescription des benzodiazépines comme coantalgiques, ou ceux de Gaudy et al. (2005), Queneau et Ostermann (2004), qui suggèrent l'utilisation de benzodiazépines dans certains types de douleurs et dans les syndromes extrapyramidaux.

\section{Conclusion}

Les trois produits testés ne sont pas toxiques aux doses thérapeutiques et semblent exercer une action analgésique moyenne visà-vis de la douleur induite par l'injection intrapéritonéale d'acide acétique, surtout pour les composés 1 et 3 , par rapport à l'acide acétylsalicylique, alors que le produit 2 a une action analgésique nettement moins importante.

\section{REMERCIEMENTS}

Ce travail a été soutenu par l'Agence Marocaine de Coopération Internationale (AMCI) que nous remercions vivement.

\section{REFERENCES BIBLIOGRAPHIQUES}

Ahabchane NH, Essassi EM. 1999. Les 1,5Benzodiazépine-2-Ones précurseurs de divers systèmes hétérocycliques à caractère pharmacologique potentiel, $J$ Soc. Chim. de Tunisie, 1: 753.

Anonyme. 1996. Guide pour les Soins et l'Utilisation des Animaux de Laboratoire, Institute of Laboratory Animal Resources. National Academy Press: Washington, D.C. 
Aveline L. 2001. Cancérologie. Estem.

Beaulieu P. 2006. Pharmacologie de la Douleur. Les Presses de l'Université de Montréal: Montréal.

Boniface M, Boniface B, Cazin JC, Cazin M, Liyckx M. 1972. Méthode de calcul de la DL $_{50 .}$ Bull. Soc. Pharm., 12-33.

Broustail M. 1967. La Souris de Laboratoire et son Elevage ( $3^{\mathrm{m} e}$ edn). Vigot Frères: Paris.

Chammache M, Zellou A, Essassi EM, Cherrah Y, Hassar M. 2001. Synthèse et étude pharmacologique dérivés de la 1,4diazepine-5-one. Ann. Pharm Fr., 59: 206-210.

Cherrah Y, Alaoui K, Faouzi MYA. 2007. Travaux pratique de pharmacologie, $2^{\text {ème }}$ année de Pharmacie, Faculté de médicine et de Pharmacie, Rabat.

Dupont C. 1970. Détermination de la $\mathrm{DL}_{50}$ chez la souris, fiche technique $\mathrm{n}^{\circ}$ 6. J. Pharmacol., f: 1(3): 407-414.

Essassi EM, Fifani J. 1987. Synthèse de la 4acétonylidène-1,5 benzodiazépin-2-one. $J$ Bull. Soc. Chim. Belg., 96: 63-69.

Ernest D. Olfert DVM, Brenda M, Cross DVM, William Ann McA. 1993. Manuel sur le Soin et l'Utilisation des Animaux d'Expérimentation. Conseil Canadien de Protection des Animaux, 1.

Gaudy JF, Arreto D. 2005. Manuel d'Analgésie en Odontostomatologie ( $2^{\text {nd }}$ édition). Masson : Paris.

Graham L, Patrick, Depovere P. 2002. Chimie Pharmaceutique. De Boeck Université.

Kirkiacharian S. 1996. Guide de Chimie Thérapeutique. Ellipses : Paris.

Kuo-Hsing I, Thoshiro I. 1978. Antitumor agents, J. Med. Chem., 21(7): 698-701.
Laroche M-J, Rousselet F. 1990. Les Animaux du Laboratoire: Ethique et Bonnes Pratiques. Masson, Paris.

Laroche M-J, Fabiani P, Rousselet F. 1986. Expertise Toxicologique des Médicaments. Masson : Paris.

Lullmann H, Klaus Mohr, Albrecht Ziegler. 2001. Atlas de Poche de Pharmacologie ( $2^{\text {nd }}$ édn). Flammarion : Paris.

Muster D, Ben Slama L. 2004. Thérapeutique Médicale Buccodentaire Moyens et Méthodes. Masson : Paris.

Queneau P, Ostermann G. 2004. Le Médecin, le Malade et la Douleur. Masson : Paris.

Sidya MS. 2004. Synthèse, réactivité et étude pharmacologique de la 4-acetonylidene1,5-benzodiazépin-2-one. Thèse de doctorat. Université Mohamed V, 174p.

Tosti A, Fleckman P, Scher R, Ralph Daniel C. 2007. Onychologie Diagnostic, Traitement, Chirurgie. Masson : Paris.

Zellou A, Cherrah Y, Essassi EM, Hassar M. 1998. Synthèse et étude pharmacologique des 1, 5-benzodiazepine-2-diones et leurs dérivés alkylés, Ann. Pharm Fr., 58(4): 169-174.

Zellou A, Cherrah Y, Essassi EM, Hassar M. 1998. Synthèse et étude pharmacologique des 1, 5-benzodiazepine-2-dithiones et leurs derivés alkylés. Ann. Pharm Fr., 58(4): 175-180.

Zniber R, Essassi EM, Lamkadem A. 1991. Caractérisation, par spectrométrie de masse, de la benzoxadiazocine obtenue par irradiation de la 4-phényl-1,5benzodiazépin-2-one. Bull. Soc. Chim. Belg., 100: 277-286. 